\title{
Simple Cooperative Relaying Strategies for WiMAX Communication System
}

\author{
Simone Morosi, Dania Marabissi, Romano Fantacci, Enrico Del Re and Sara Jayousi \\ Department of Electronics and Telecommunications \\ University of Florence \\ Via S. Marta 3, 50139, Florence, Italy \\ Telephone: +39055 4796485 \\ Fax: +39055 472858 \\ Email: simone.morosi@unifi.it
}

\begin{abstract}
Cooperative Relaying Systems represent a promising solution for future wireless communications. In this paper simple cooperative relaying strategies are proposed in order to improve the performance of a WiMAX network, overcoming the drawbacks of a Non-Line-Of-Sight (NLOS) propagation. The cooperative schemes proposed combine the Amplify and Forward (AAF) and the Decode and Forward (DAF) algorithm with Delay Diversity (DD) and Cyclic Delay Diversity (CDD) techniques, in order to exploit the features of the wireless communication channel. We investigated the pros and cons of each scheme, comparing the assumptions which are required and the simulation results with respect to two cooperative implementations of the Alamouti scheme.
\end{abstract}

\section{INTRODUCTION}

The IEEE802.16 standard [1] is a technology which has been proposed for the "wireless" broadband communications: it can be considered as a viable alternative to the wired backhaul and to the last miles deployments that uses cable modems, DSL and optical carrier technologies. The WiMAX standard defines the technical features of this communication system. The physical layer of the Mobile WiMAX system resorts to the Orthogonal Frequency Division Multiple Access (OFDMA) to cope with the drawbacks of the Non-LineOf-Sight (NLOS) propagation; moreover, several schemes of modulation and coding rate, that can be used depending on channel and interference conditions, are defined in order to achieve the highest spectral efficiency. Finally, to provide a higher throughput in a multipath environments and increase the link range, some multiple antenna options are supported by the standard. However, the transmit diversity techniques require more than one antenna at the transmitter side and could not be used because of cost, size and hardware complexity constrains [2].

To exploit the advantages of MIMO system in these networks, the cooperative communication systems can be introduced. The main idea of this class of communication schemes is that every node can share its antenna in order to create a virtual MIMO system and increase the quality of service by cooperation: in particular we focus on two affordable

This work has been supported by Italian Research Program (PRIN 2007) Satellite-Assisted Locallzation and Communication system for Emergency services (SALICE). cooperative relaying strategies, which can be used in order to improve the performance of a WiMAX network.

The deployment of the Relay Stations, as defined in IEEE802.16j [3], can be seen a promising solution for future wireless communication. The cooperative relaying systems can be classified in two main categories: non-regenerative and regenerative; the difference between these schemes depends on the operations which are implemented at the relay station. In the non-regenerative systems the relay acts according to the Amplify and Forward algorithm, while in the regenerative ones the relay applies the rules of the Decode and Forward algorithm. Considering these two cooperative schemes as principle schemes, the purpose of this paper is to introduce Delay Diversity (DD) and Cyclic Delay Diversity (CDD) techniques in the context of cooperative communications OFDMbased networks: in particular, combining regenerative and nonregenerative systems with DD and CDD schemes allows to exploit the features of the wireless communication channel; in fact these systems take advantages from an increasing of the channel frequency selectivity which makes them very suitable for the NLOS environment, that is one of the main cause of the performance loss. Besides the simplicity of these cooperation schemes makes them very cost-effective.

In the following the organization of the paper is described: in Section II we analyse the two cooperative schemes, namely the Cooperative Delay and Cyclic Delay Diversity, which are compared with the cooperative versions of the Alamouti STBC and SFBC schemes. Section III describes the cooperation scenario and the specifications adopted in the simulations, whose results are shown in Section IV.

\section{Cooperative Relaying Strategies}

The cooperative relaying network which is considered in this paper is based on three single-antenna equipped nodes: the Source node (S), the Destination node (D) and CooperativeRelay node (R). In this network each node (fixed or mobile) can play the role of both the destination and the relay so that two independently faded versions of the transmitted signal arrive at D: one directly from $\mathrm{S}$ and the other one through $\mathrm{R}$. The transmission of the same information data from different locations affords spatial diversity at the receiver. As it is 


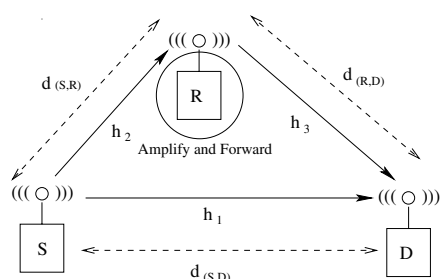

Fig. 1. Cooperative Delay Diversity theoretical scheme

known, the cooperative-node can process the received signal according to two different techniques:

- Amplify and Forward: the signal is amplified and retransmitted.

- Decode and Forward: the signal is decoded and retransmitted after being re-modulated.

The drawback of the first method is that even noise and interference are amplified, while in the second one error propagation represents the main loss of the algorithm.

In the following subsections we analyse how the DD and CDD schemes can be adopted in a cooperative system, highlighting the advantages and disadvantages of each scheme; moreover, we compare them with a cooperative implementation of the Alamouti scheme.

\section{A. Cooperative Delay Diversity scheme}

The DD technique is based on the transmission of one or more delayed copies of the same information data in order to increase the delay spread of the channel, and, therefore the frequency selectivity. In a cooperative network the transmitted signal arrives at the destination node through two different paths, which are characterized by different delays:

1) $\delta_{0}$ is the propagation delay of the signal through the direct path (from $S$ to $D$ );

2) $\delta_{1}$ is the propagation delay of the signal through indirect path (from $\mathrm{S}$ to $\mathrm{D}$ through $\mathrm{R}$ );

The delay $(\delta)$ of the DD scheme is the difference between these two delays:

$$
\delta=\delta_{1}-\delta_{0}=\frac{d_{(S, R)}+d_{(R, D)}}{c}-\frac{d_{(S, D)}}{c}
$$

where $d_{(i, j)}$ denotes the distance between the node $i$ and the node $j$ and $c$ represents the speed of light.

Therefore, the DD technique can be seen as a natural cooperative-relaying transmission: in fact no additional operation is required at the destination node. In order to keep the complexity low, the relay node can act according to the Amplify and Forward algorithm, amplifying and retransmitting the received signal (Fig.1).

In the theoretical scheme the relay node is assumed to be transparent: nonetheless, since the relay node can not simultaneously receive and transmit on the same frequency, a Time Division Multiplex mode is assumed for the relay. Therefore, we propose two different cooperative schemes (Fig.2) which are characterized by a two phase protocol:

- $\underline{\text { Scheme-1 }}$

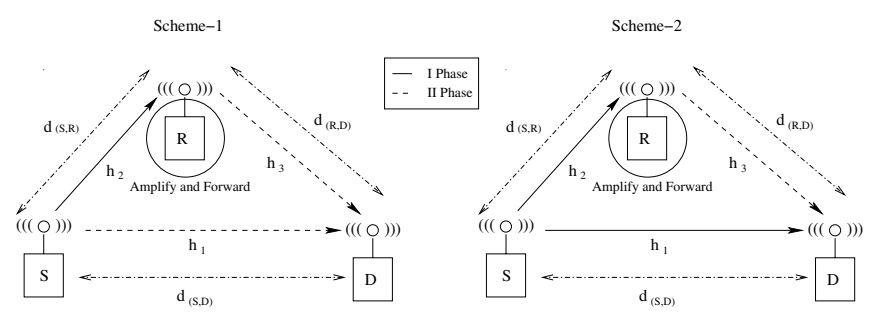

Fig. 2. Cooperative Delay Diversity practical schemes

I Phase: $\mathrm{S}$ node transmits the signal to $\mathrm{R}$;

II Phase: $\mathrm{S}$ and $\mathrm{R}$ transmit the original and the previously received signal, respectively, to $D$.

In this scheme two different delayed copies of the original signal arrive at $\mathrm{D}$. In particular the natural delay is originated by the difference between the path from $\mathrm{S}$ to $\mathrm{D}$ and the one from $\mathrm{R}$ to $\mathrm{D}$ :

$$
\delta=\left|\frac{d_{(S, D)}}{c}-\frac{d_{(R, D)}}{c}\right|
$$

Therefore, no additional operation is implemented at $\mathrm{R}$ and no synchronization is needed at $\mathrm{D}$ between the received signals: as a result, the scheme is very simple and cost effective.

- Scheme-2

I Phase: $\mathrm{S}$ transmits the signal both to $\mathrm{D}$ and $\mathrm{R}$;

II Phase: $\mathrm{S}$ is idle and $\mathrm{R}$ transmits the previously received signal to $\mathrm{D}$.

In this scheme $\mathrm{R}$ behaves as in the Scheme- 1 and the delay is still given by the expression (2). However, in order to properly combine the signals coming from $S$ and $R$, a buffer is needed at $\mathrm{D}$ to store the signal received from $\mathrm{S}$ in the first phase.

\section{B. Cooperative Cyclic Delay Diversity scheme}

Adapting the CDD schemes to the cooperative relaying systems is more complicated than DD ones: in fact, in the CDD techniques, the delay, which is distinctive of each transmitted copy of the signal, is replaced with a cyclic shift, cyclic delay, performed before the insertion of the cyclic prefix of the OFDM system. As it is known [4], the transmission of different cyclically shifted copies of the original signal permits to obtain the same effect of the DD technique.

In this scheme the relay node follows the Decode and Forward algorithm, by demodulating and re-modulating the received signal and performing the cyclic shift, before inserting a cyclic prefix and up converting to the RF-band.

As in the DD scheme, in order to adopt the CDD scheme in a cooperative network we have to assume that the system works in a Time Division Multiplex mode. Therefore, a data packet transmission consists of two phases, which can be implemented according to two different cooperative procedures (Fig.3):

\section{- Scheme-1}

I Phase: $\mathrm{S}$ transmits the signal both to $\mathrm{D}$ and $\mathrm{R}$; II Phase: $\mathrm{S}$ is idle and $\mathrm{R}$ transmits the shifted signal to $\mathrm{D}$. 


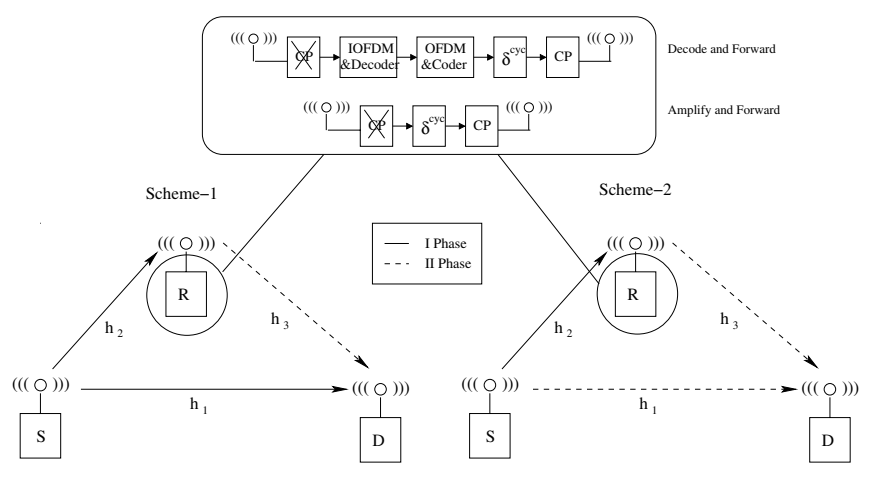

Fig. 3. Cooperative Cyclic Delay Diversity practical schemes

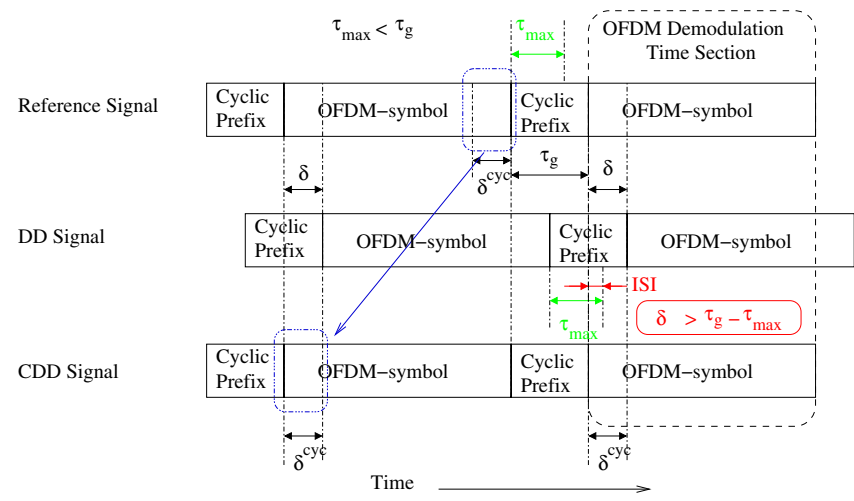

Fig. 4. Comparison between CDD and DD techniques

- $\underline{\text { Scheme-2 }}$

I Phase: $\mathrm{S}$ transmits the signal to $\mathrm{R}$;

II Phase: $\mathrm{S}$ and $\mathrm{R}$ transmit the original and the shifted signal to $\mathrm{D}$, respectively.

In both schemes a buffer is needed at the Destination node in order to synchronize the different versions of the OFDM symbols coming from $\mathrm{S}$ and $\mathrm{R}$.

Comparison between Delay and Cyclic Delay Diversity:

The difference between DD and CDD are pointed out in Fig. 4 which represents the transmission of two consecutive OFDM symbols: in particular, we can note:

- the Reference Signal, which represents the original signal or the signal taken as a reference;

- the DD and CDD Signal, which are respectively the delayed and the cyclic-delayed copy of the original signal.

The DD-signal is a delayed copy of the original signal so that, at the receiver, there is the superimposition of the first OFDM symbol of the DD-signal and of the cyclic prefix of the second OFDM symbol of the Reference-signal. Therefore, in order to avoid the ISI the delay spread of the propagation channel is to be taken into account. The following condition guarantees an ISI-free signal at the receiver in DD scheme:

$$
\delta \leq \tau_{g}-\tau_{\max }
$$

where $\tau_{g}$ represents the duration of the cyclic prefix and $\tau_{\max }$ denotes the delay spread of the channel. Assuming the guard interval as constant, the range of possible delays is strongly limited; this disadvantage can be overcome by the CDD technique [5] [6], in which the signal is not delayed, but just cyclically shifted. Hence, the choice of the cyclic delay does not depend on the cyclic prefix length and a longer cyclic delay does not involve any extension of the cyclic prefix and a consequent capacity loss (as it is in the DD scheme). However, in order to achieve a superimposition of the signals within the assigned bandwidth of the $N$ subcarriers of the OFDM symbol, both the delay and the cyclic delay have to fulfill the following condition:

$$
\delta \geq \frac{1}{B} \quad B=\frac{N}{T_{S}}
$$

where $B$ is the bandwidth and $T_{S}$ is the sampling rate of the transmitted signal.

Optimization: Under the assumption (4) (for both DD and $\mathrm{CDD}$ ) and (3) (for DD), the choice of the optimum delay depends both on the number of cooperative nodes and the number of the subcarriers; moreover, since each delay can be seen as a specific phase rotation, it also depends on the modulation alphabet [7]. In [8] two delay optimization schemes are introduced, but both of them require channel feedback information from D to the relay nodes. However we have found the optimal value through simulations.

Delay and Cyclic Delay Diversity's effects: We have already observed that a cyclic shift has the same effect as a real delay. Therefore at the receiver, after removing the cyclic prefix, the DD and the CDD schemes yield the same signal (assuming $\left.\delta=\delta^{c y c}\right)$.

The DD and CDD effects can be analysed also in terms of channel transfer function and channel impulse response. In fact, as demostrated in [9] transmitting delayed or cyclically shifted copies of the same signal through a propagation channel is equivalent to transmitting the reference signal through a more frequency-selective channel. Therefore, we can conclude that the MISO channel (Multiple-Input Single-Output) is transformed into a SISO (Single-Input Single-Output) channel with increased frequency selectivity and the spatial diversity is transformed into frequency diversity. The increase of the frequency selectivity makes the error distribution changes: this feature can be exploited by the use of FEC encoder and decoder with a considerably improvement of the coding gain.

In a cooperative system with $N_{c}$ relay nodes and an errorfree transmission between $\mathrm{S}$ and the relay nodes, the equivalent transfer function $H_{e q u}(l)$, which includes the DD or CDD effects, can be written as:

$$
H_{e q u}(l)=\sqrt{\frac{N}{N_{c}+1}} \sum_{i=0}^{N_{c}} H_{i}(l) e^{-j \frac{2 \pi}{N} \delta_{i} l}
$$

where $H_{i}(l)$ represents the frequency domain channel fading coefficients for $l$-th subcarrier of the transmitted OFDM symbol from Relay node- $i$ to the Destination node and $\delta_{i}$ denotes the delay or the cyclic delay associated to the Relay node- $i$ (the source is seen as the relay node- 0 with $\delta_{0}=0$ ). Therefore, in the time domain the equivalent channel impulse 
response ends up being the superposition of the $N_{c}$ channel impulse responses of the channels between the relay node- $i$ and $\mathrm{D}$, each shifted in time by the specific $\delta_{i}$ and the channel impulse response of the channel between $\mathrm{S}$ and $\mathrm{D}$.

\section{Cooperative Alamouti scheme}

We have also extended the classical Alamouti STBC scheme [10] to a cooperative system as a term of reference of the schemes described in the previous subsections. In OFDM system Alamouti scheme can be implemented both in the time domain (STBC, Space Time Block Coding) and in the frequency domain (SFBC, Space Frequency Block Coding) [11] [12] [13]. The Alamouti scheme which has been considered involves two Tx-antenna and one Rx-antenna, achieves the full spatial diversity and is based on the following procedures:

- STBC: different versions of consecutive OFDM symbols are transmitted from the antennas every two OFDM symbol duration.

- SFBC: different OFDM symbol, results of combining the constellation map symbols every two adjacent subcarriers are transmitted from the antennas.

At the receiver end, after being combined, the signals are input to a maximum likelihood detector. The channel coefficients are required to be constant: for the STBC-scheme, they must remain constant during two consecutive OFDM symbols, in time domain, while, for SFBC-scheme across two adjacent subcarriers, in frequency domain.

To introduce an Alamouti scheme in a cooperative system, the two Tx-antenna are assumed as cooperative nodes, which know all the information to be transmitted. Therefore, in a starting phase, the source $\mathrm{S}$ has to transmit the information to both the cooperative nodes .

All the other cooperation schemes which can be proposed are more complex than the DD and CDD cooperative schemes and require the presence of a combiner at the destination node. Besides, the assumptions made for the channel propagation model are not always realistic.

\section{Working CONDITIONS: ApPLICATION to Mobile WIMAX}

The cooperative schemes which have been described in the previous section are applied to a WiMAX network. In order to increase the diversity gain and improve the performance, the IEEE802.16 standard supports several multiple antenna options as Space Time Codes (STC), Multiple-Input MultipleOutput antenna system and Adaptive Antenna systems (AAS) [14]. The cooperative schemes are a promising solution to further improve the performance in term of Bit Error Rate, Block Error Rate and outage probability of a WiMAX network. Besides they can be used to increase coverage and to enhance throughput and system capacity.

\section{A. An hypothetical scenario}

The adopted cooperation scenario is represented in Fig.5. The two Subscriber Stations (SS) represents the source and the

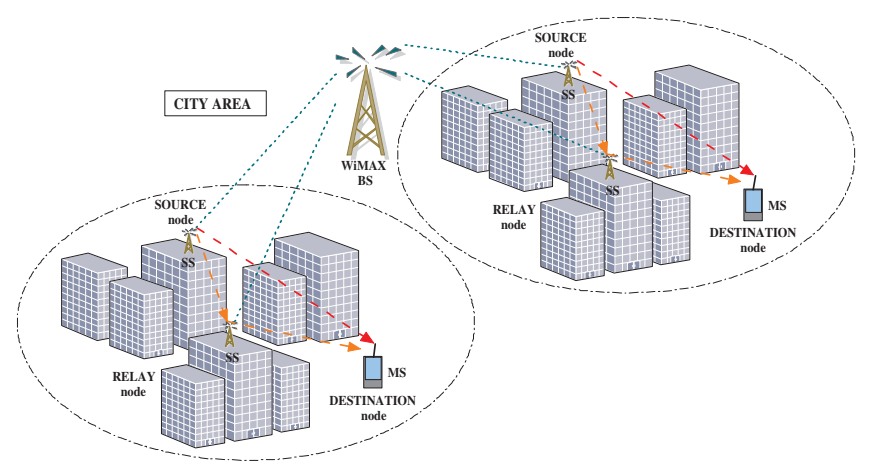

Fig. 5. Downlink WiMAX Cooperative scenario

relay node while the Mobile Station (MS) is the destination node.

According to the Mobile WIMAX specifications, an OFDMA system is implemented at the transmitter side and a cyclic prefix is introduced to avoid ISI at the receiver. The system includes:

- Modulation schemes: QPSK, 16 QAM and 64 QAM.

- Channel coding: $(171,133)_{\text {oct }}$ convolutional code with constraint length $K=7$ and code rate $1 / 2$.

- Block interleaver: two permutations are defined. The former makes the adjacent coded bits mapped onto non adjacent subcarriers, realising an interleaving in the frequency domain; the latter guarantees that adjacent coded bits are mapped alternately onto less or more significant bits of the constellation in order to avoid long runs of lowly reliable bits. In the simulation we consider two different block-size of coded bits: 256 and 2560 .

The channel bandwidth is equal to $10 \mathrm{MHz}$ and the FFT size is 1024. The OFDMA symbol duration is equal to 102.9 $\mu_{s}$ and the cyclic prefix is assumed equal to $11.4 \mu_{s}$. At the receiver after the cyclic prefix removal, the Zero Forcing equalization is performed, assuming the knowledge of channel state information. The OFDM demodulation is followed by the deinterleaver and the convolutional decoder (hard-decision Viterbi Decoder). The channel model which has been used for the simulation has been derived according to ITU Reccomandation for the $3 \mathrm{G}$ cellular system. In particular, we assume a multipath Rayleigh fading channel with non-line-of-sight (NLOS) propagation and the power-delay profile of the ITU Vehicular A channel. The Mobile Speed is $60 \mathrm{~km} / \mathrm{h}$.

In the DD and CDD cooperative schemes, besides the channel state information, the node $\mathrm{D}$ also needs to know respectively the delay and cyclic delay to perform the channel equalization. While $\delta^{c y c}$ can be transmitted as additional information by $\mathrm{R}, \delta$ can be derived by $\mathrm{D}$ through distance information: in fact, D knows its distance both from $\mathrm{S}$ and $\mathrm{R}$.

In the simulations we also assume:

- Cooperative DD The noise introduced by $\mathrm{R}$ is negligible and no amplification is performed. Therefore, $\mathrm{R}$ can be seen as a transparent node.

- Cooperative CDD We consider an error-free transmission 
between $\mathrm{S}$ and $\mathrm{R}$, assuming that the noise introduced by $\mathrm{R}$ is negligible.

\section{Simulation Results}

The performance of the proposed systems has been verified by simulations. The cooperative and non-cooperative WiMAX systems are compared, assuming the same total transmitted power.

In Fig.6 and in Fig.7, respectively, the Cooperative DD and CDD schemes are compared with the non cooperative scheme. For all the modulations, which have been considered, a remarkable improvement is achieved $\left(6-7 \mathrm{~dB}\right.$ for $\left.\mathrm{BER}=10^{-3}\right)$. The optimum $\delta$ and the $\delta^{c y c}$ values have been determined by simulations. According to the working conditions adopted, the $\delta_{o p t}$ is equal to the $\delta_{\max }$ and corresponds, in the theoretical scheme, to a linear distance of $2,7 \mathrm{Km}$ as the difference between the direct and the reflected paths.

In order to determinate $\delta_{o p t}$ and $\delta_{o p t}^{c y c}$ we analyse the BER performance for different SNRs and delays for a QPSK modulated signal. As we can see for CDD scheme Fig.8, the optimum performance is achieved for values of $\delta_{\text {opt }}^{c y c}$ included in the range $[128,450]$ samples: note that the BER curves is characterized by a near-zero slope within this range. For what concerns the DD scheme, as previously mentioned, the effects on the signals are the same of the CDD architecture: however, in this scheme the admissible range for delay values is limited by $\delta_{\max }$ which is equal to 100 samples in order to guarantee the condition (3). Therefore, the optimum performance is reduced in comparison with the CDD scheme: as indicated by Fig.8, the $\delta_{o p t}$ value is equal to the $\delta_{\max }$.

Finally, in Fig.9, Fig.10 and Fig.11 we compare the BER performance of all the cooperative schemes for the coded and the interleaved systems (16 QAM modulated). We observe that even if the performance of the Cooperative Alamouti schemes overcomes both the Cooperative DD and CDD ones, the improvement is marginal both in the coded and the 256-block interleaved cases. On the contrary, with 2560-block interleaving the Alamouti solutions gains $2-3 \mathrm{~dB}$ (for $\mathrm{BER}=10^{-3}$ ), as shown in Fig.11. It is worth recalling that both the Cooperative Alamouti schemes are more complex in comparison than the Cooperative DD and CDD ones. The promosing results of the Cooperative DD and CDD schemes claim for a further analysis.

\section{CONCLUSIONS}

In this paper simple cooperative relaying strategies for a WiMAX network are proposed in order to improve the performance of the system with one cooperative node. The Amplify and Forward and Decode and Forward algorithms are combined with DD and CDD techniques with the goal of reduce the impairments which is caused by the wireless communication channel. All the investigated cooperative schemes are characterized by a significant gain with respect to the non-cooperative ones. Besides, the results show that the performance in term of Bit Error Rate of the Cooperative DD and CDD are almost the same of the Cooperative Alamouti schemes, except in the case of 2560-block interleaved systems. However, the Cooperative Alamouti schemes require strong assumptions on the channel propagation model and need the presence of the combiner at the Destination node. On the contrary, the DD and CDD cooperative schemes represent two simple promising solutions to improve the performance of the system without any additional complexity requirement.

\section{REFERENCES}

[1] W. Forum, "Mobile wimax part i: A technical overview and performance evaluation," WiMAX Forum, Tech. Rep., 2006.

[2] A. Nosratinia, T. E. Hunter, and A. Hedayat, "Cooperative communications in wireless networks," IEEE Communication Magazine, vol. 42, pp. 74-80, Oct. 2004.

[3] Baseline Document for Draft Standard 802.16j-2006. Part 16: Air Interface for Fixed and Mobile Broadband Wireless Access Systems.Multihop Relay Specification, Std. 802.16j-06/026r2, 2007.

[4] Y. Zhang, J. Cosmas, and Y. Song, "Future transmitter/receiver diversity schemes in broadcast wireless networks," IEEE Communications Magazine, vol. 44, pp. 120-127, Oct. 2006.

[5] S. Plass and A. Dammann, "Cellular cyclic delay diversity for next generation mobile systems," VTC2006, pp. 1-5, 2006.

[6] Y. Zhang, J. Cosmas, M. Bard, and Y.-H. Song, "Diversity gain for dvb-h by using transmitter/receiver cyclic delay diversity," IEEE Transactions on Broadcasting, vol. 52, pp. 464-474, Dec. 2006.

[7] M. Bossert, A. Huebner, F. Schuehlein, H. Hass, and E. Costa, "On cyclic delay diversity in ofdm based transmision schemes," InOWo 2002, vol. 16.

[8] B. Slimane, X. Li, B. Zhou, N. Syed, and M. Dheim, "Delay optimization in cooperative relaying with cyclic delay diversity," in IEEE International Conference on Communication (ICC'08), May 2008, pp. $3553-3557$.

[9] A. Dammann and S. Plass, "Cyclic delay diversity: Effective channel properties and applications," in Proc.of the WWRF17-WG4-06.

[10] S. M. Alamouti, "A simple transmit diversity technique for wireless communications," IEEE J. Select Areas in Communications, vol. 16, pp. 1451-1458, Oct. 1998.

[11] K. F. Lee and D. B.Williams, "A space-frequency transmitter diversity technique for ofdm systems," IEEE Globecom 2000, vol. 3.

[12] R.U.Nabar, H.Bolcskei, and F.W.Kneubuhler, "Fading relay channels: Performance limits and space-time signal design," IEEE J.Select. Areas Communications, vol. 22, pp. 1099-1109, Aug. 2004.

[13] A. Lodhi, F. Said, M. Dohler, and H. Aghvami, "Performance comparison of space-time block coded and cyclic delay diversity mc-cdma systems," IEEE Wireless Communications, vol. 12, pp. 38-45, Apr. 2004.

[14] M. Gidlund, "On wimax performance with multiple antenna transmission," in IEEE Int.Conf.ICCE, Jan. 2007, pp. 1-2.

[15] S. B. Slimane and A. Osseiran, "Relay communication with delay diversity for future communication systems," VTC 2006, pp. 1-5, 2006.

[16] A. Osseiran, A. Logothetis, S. B. Slimane, and P. Larsson, "Relay cyclic delay diversity: Modeling and system performance," in IEEE International Conf.on Signal Processing Communication, Nov. 2007.

[17] A. Antó, "Cyclic delay diversity in cooperative communications and single hop systems," M. Eng. thesis 2007, KTH School of Information and Communications Technology.

[18] IEEE Standard for Local and metropolitan area networks. Part 16: Air Interface for Fixed and Mobile Broadband Wireless Access Systems, IEEE Std. 802.16e-2005, 2006. 


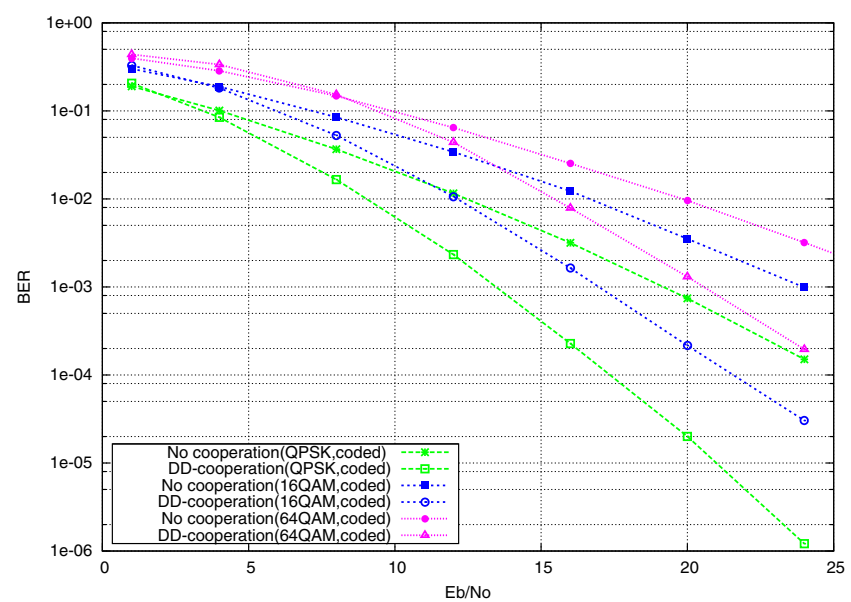

Fig. 6. Performance comparison of WiMAX system without cooperation and Cooperative DD with $\delta_{\text {opt }}=100$ samples.

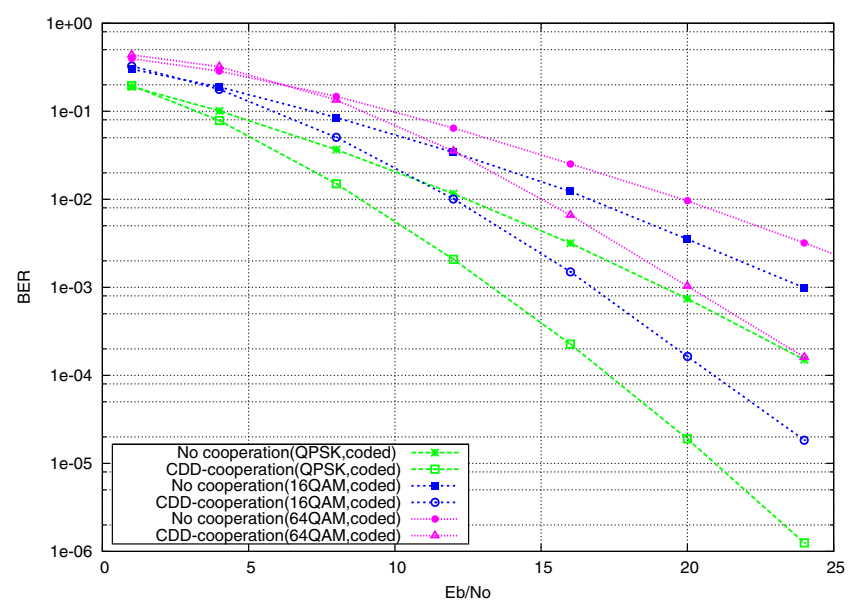

Fig. 7. Performance comparison of WiMAX system without cooperation and Cooperative CDD with $\delta_{o p t}^{c y c}=128$ samples.

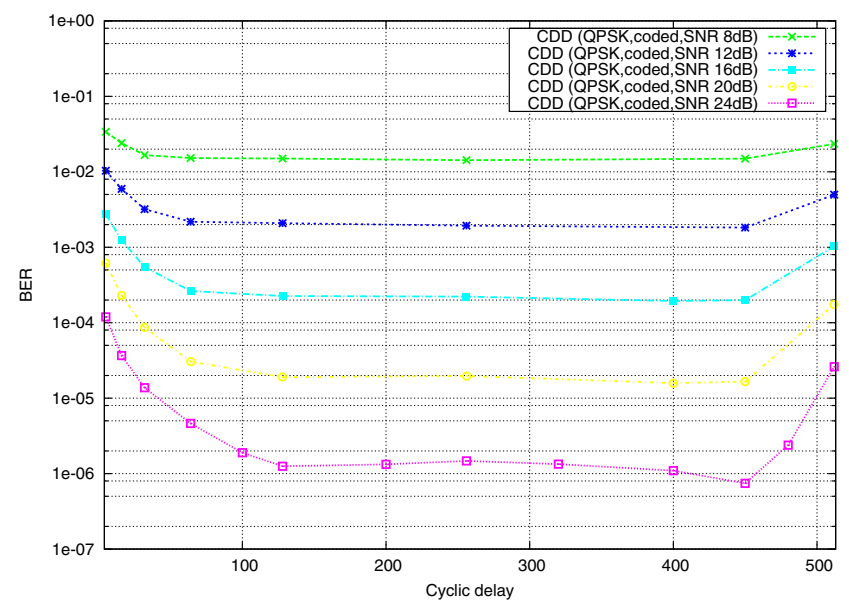

Fig. 8. Delay and cyclic delay BER-optimization.

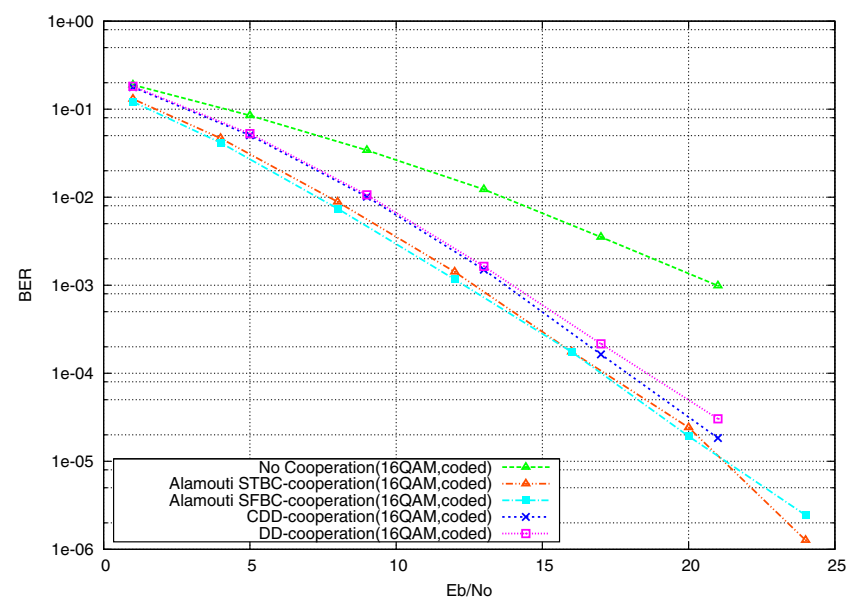

Fig. 9. Performance comparison of WiMAX coded-system without cooperation and with Cooperative DD $\left(\delta_{o p t}=100\right.$ samples $), \operatorname{CDD}\left(\delta_{o p t}^{c y c}=128\right.$ samples) and Alamouti STBC and SFBC.

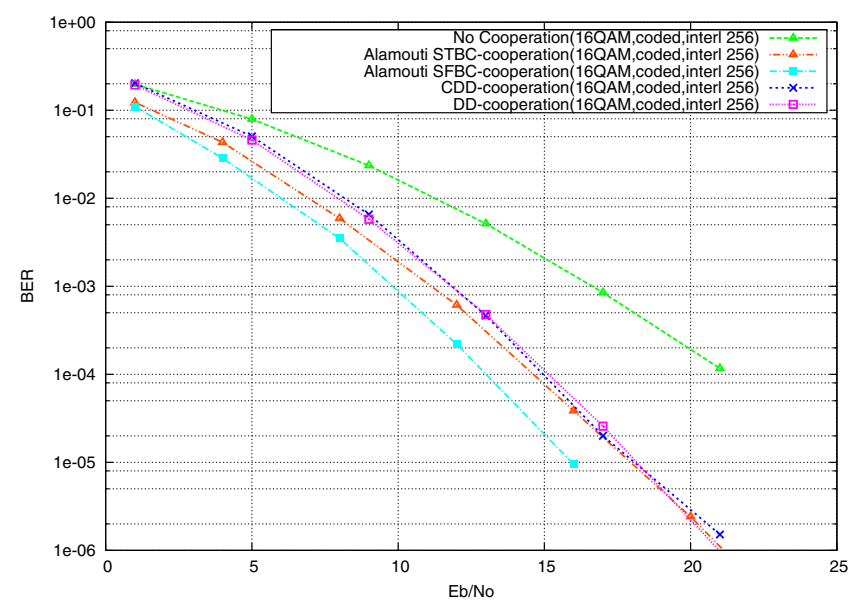

Fig. 10. Performance comparison of WiMAX coded and 256-block interleaved system without cooperation and with Cooperative DD $\left(\delta_{\text {opt }}=64\right.$ samples), CDD ( $\delta_{\text {opt }}^{c y c}=64$ samples $)$, Alamouti STBC and SFBC.

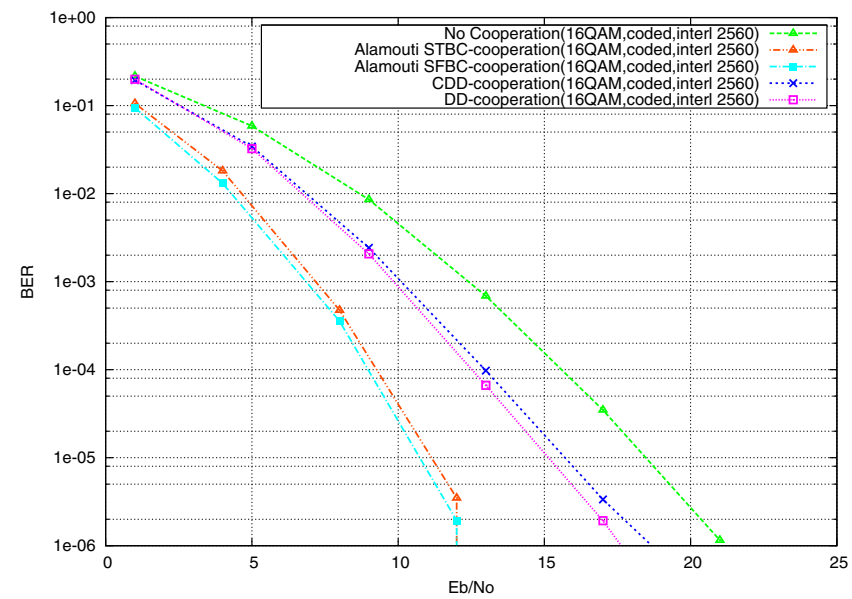

Fig. 11. Performance comparison of WiMAX coded and 2560-block interleaved system without cooperation and with Cooperative DD $\left(\delta_{\text {opt }}=32\right.$ samples), CDD ( $\delta_{\text {opt }}^{\text {cyc }}=32$ samples), Alamouti STBC and SFBC. 